\title{
Microscopic description of the twist mode in normal and superfluid trapped Fermi gases
}

\author{
Marcella Grasso, ${ }^{1,2}$ Michael Urban, ${ }^{2}$ and Xavier Viñas ${ }^{3}$ \\ ${ }^{1}$ Dipartimento di Fisica e Astronomia and INFN, Via Santa Sofia 64, I-95123 Catania, Italy \\ ${ }^{2}$ Institut de Physique Nucléaire, 15 rue Georges Clémenceau, F-91406 Orsay Cedex, France \\ ${ }^{3}$ Departament d'Estructura i Constituents de la Matèria, Facultat de Fìsica, Universitat de Barcelona, \\ Diagonal 647, E-08028 Barcelona, Spain \\ (Received 19 July 2004; revised manuscript received 28 September 2004; published 19 January 2005)
}

\begin{abstract}
We investigate the "twist" mode (rotation of the upper against the lower hemisphere) of a dilute atomic Fermi gas in a spherical trap. The normal and superfluid phases are considered. The linear response to this external perturbation is calculated within the microscopic Hartree-Fock-Bogoliubov approach. In the normal phase the excitation spectrum is concentrated in a rather narrow peak very close to the trapping frequency. In the superfluid phase the strength starts to be damped and fragmented and the collectivity of the mode is progressively lost when the temperature decreases. In the weak-pairing regime some reminiscence of the collective motion still exists, whereas in the strong-pairing regime the twist mode is completely washed out. The disappearance of the twist mode in the strong-pairing regime with decreasing temperature is interpreted in the framework of the two-fluid model.
\end{abstract}

DOI: 10.1103/PhysRevA.71.013603

PACS number(s): 03.75.Ss, 21.60.Jz

\section{INTRODUCTION}

The experimental and theoretical development of BoseEinstein condensation of trapped bosonic atoms [1] has also triggered the investigation of trapped atomic Fermi gases at very low temperature [2]. One of the main goals in the research of these Fermi systems is to detect the transition from the normal to the superfluid phase, associated with the appearance of a macroscopic order parameter of strongly correlated Cooper pairs below a certain critical temperature $T_{c}$. In order to have an attractive $s$-wave interaction which can provide the pairing correlations, the atoms must be trapped and cooled in two different hyperfine states as has been achieved in several recent experiments (see, e.g., Ref. [3]). From the theoretical side, the pairing problem of trapped fermions has been studied from different points of view [4-6].

Besides the ground-state properties, there is also interest in knowing the spectrum of collective excitations. As we stated above, ultracold atomic Fermi gases are assumed to become superfluid below $T_{c}$, and it is therefore important to study low-energy collective modes also in the superfluid phase $[7,8]$. Having different properties in the normal and superfluid phases, these excitations can serve as experimental signals for superfluidity. For instance, the frequencies of breathing modes of trapped atomic Fermi gases measured in recent experiments [9] give strong indications that the superfluid phase has been reached.

It is interesting to compare the situation of trapped fermionic atoms to that of atomic nuclei, which can also show a superfluid behavior. Contrary to the nuclear case, the fact that the interaction in atomic gases is tunable experimentally allows one to study the collective modes in different regimes. For dilute systems, the atom-atom interaction can be parametrized by a zero-range force proportional to the $s$-wave scattering length between atoms in two hyperfine states $[6,8]$. By changing the applied magnetic field around a Feshbach resonance [10], the $s$-wave scattering length can be modified. In one limiting regime, that of weak pairing, which is similar to the situation in atomic nuclei, the pairing results only in a small perturbation to the response of the system to the external probe. In the other limit of strong pairing the response is dominated by the effects of superfluidity.

Many of the collective excitations show features proper to Landau's zero-sound modes in bulk Fermi liquids [11] which for finite Fermi systems translate into modes analogous to those of an elastic body [12]. Since the trapped atomic Fermi gases contain a very large number of atoms, the singleparticle orbital angular momenta near the Fermi surface can also become very large. Consequently, important orbital effects such as excitations having angular momentum and parity $J^{P}=1^{+}$and $2^{-}$will exist, which correspond to magnetic resonances of $M 1$ or $M 2$ type, respectively, in atomic nuclei. The $2^{-}$excitation is the so-called twist mode, in analogy to the quadrupole torsional vibration of an elastic sphere $[12,13]$. From a macroscopic point of view, the twist consists of a coherent counterrotation of the particles in the upper hemisphere against those in the lower hemisphere. For small amplitudes, it corresponds to a purely kinetic excitation without spatial distortion of the equilibrium shape.

The twist mode has been studied in different Fermi systems. In nuclei, this mode has been analyzed from a semiclassical point of view within a fluid-dynamical description [13]. From a quantum-mechanical point of view, this mode has been studied so far only for magic nuclei (i.e., without pairing) such as ${ }^{90} \mathrm{Zr}$ and ${ }^{208} \mathrm{~Pb}[14,15]$. More recently, some experimental effort has been made to detect this mode by backward inelastic electron scattering [16]. A direct evidence for the existence of the orbital twist mode (to be distinguished from the $2^{-}$spin-flip mode) in nuclei has been achieved by comparing electron and proton scattering cross sections of ${ }^{58} \mathrm{Ni}$ [17]. The twist mode has also been theoretically studied in metallic clusters [18] although it has not yet been detected.

So far, the theoretical study of the twist mode in trapped atomic Fermi gases has been done in the hydrodynamical 
description and in the normal phase only [19]. In the case of an $s$-wave interaction, a moderate shift of the twist frequency of about $10 \%$ with respect to the noninteracting case was found, which is consistent with the fact that for a transverse zero sound the $s$-wave interaction does not contribute to the restoring force $[14,20]$.

In the present article, our aim is different. We will analyze the effect of pairing correlations on the twist mode. This effect has not been considered in any of the theoretical studies mentioned above, either for atomic nucleior for metallic clusters or trapped Fermi gases. Of particular interest can be the study of the strong-pairing regime, because it is known that in this case the low-energy collective modes are strongly affected by the pairing and can become signatures that the superfluid phase is reached $[7,8]$.

The paper is organized as follows. In Sec. II, we sketch the derivation of the twist response function in the superfluid phase, using a Hartree-Fock-Bogoliubov or Bogoliubov-de Gennes framework [21,22]. In Sec. III, we consider the twist mode in the normal phase within a quantum-mechanical description. Section IV is devoted to the study of the twist mode in the superfluid phase in the cases of weak- and strong-pairing correlations. Finally, our conclusions are given in the last section.

\section{QUASIPARTICLE RESPONSE FUNCTION}

In this article we will consider an atomic Fermi gas (atomic mass $m$ ), trapped in a spherical harmonic trap with frequency $\Omega$. We assume that the atoms equally occupy two hyperfine states, denoted by $\sigma= \pm 1$. Because of the low density of the gas, the interaction between the atoms can be regarded as pointlike and its strength can be parametrized by the $s$-wave atom-atom scattering length $a$. In order to simplify the notation, we will express all quantities in harmonic oscillator (HO) units, i.e., frequencies in units of $\Omega$, energies in units of $\hbar \Omega$, temperatures in units of $\hbar \Omega / k_{B}$, and lengths in units of the oscillator length $l_{H O}=\sqrt{\hbar /(m \Omega)}$. Furthermore, instead of the scattering length we will use the coupling constant $g=4 \pi a / l_{H O}$ as the parameter of the interaction strength.

The twist is a motion where the upper and lower hemispheres rotate in the opposite sense back and forth around the $z$ axis with an angle proportional to $z$. This mode can be excited in both spherical and deformed (with a rotation axis) systems. Such a motion can be generated by the operator $z L_{z}$, where $L_{z}=-i\left(x \boldsymbol{\nabla}_{y}-y \boldsymbol{\nabla}_{x}\right)$ denotes the $z$ component of the angular momentum operator. Restricting our description to small amplitudes, we can use linear response theory in order to treat the oscillations around equilibrium. Then the main problem consists in calculating the equilibrium state. In order to describe the system in the superfluid phase, this is done within the framework of a Hartree-Fock-Bogoliubov (HFB) or Bogoliubov-de Gennes [21,22] calculation similar to that presented in Ref. [6], but with the modified regularization scheme for the gap equation described in Refs. [23,24]. We refer to Ref. [24] for more details about our approach. The calculation provides the wave functions $u_{\alpha}(\boldsymbol{r})$ and $v_{\alpha}(\boldsymbol{r})$ satisfying the HFB equations,

$$
\begin{aligned}
& {\left[H_{0}+W(\boldsymbol{r})\right] u_{\alpha}(\boldsymbol{r})+\Delta(\boldsymbol{r}) v_{\alpha}(\boldsymbol{r})=E_{\alpha} u_{\alpha}(\boldsymbol{r}),} \\
& \Delta(\boldsymbol{r}) u_{\alpha}(\boldsymbol{r})-\left[H_{0}+W(\boldsymbol{r})\right] v_{\alpha}(\boldsymbol{r})=E_{\alpha} v_{\alpha}(\boldsymbol{r}) .
\end{aligned}
$$

Here $H_{0}$ denotes the Hamiltonian of the noninteracting HO minus the chemical potential, $H_{0}=\left(-\nabla^{2}+r^{2}\right) / 2-\mu$, while the interaction is accounted for in a self-consistent way through the Hartree potential $W(\boldsymbol{r})$ and the pairing field $\Delta(\boldsymbol{r})$.

Now let us consider the retarded correlation function

$$
\Pi_{0}(\omega)=-i \int_{0}^{\infty} d t e^{i \omega t}\langle\langle[Q(t), Q(0)]\rangle\rangle,
$$

where $\langle\langle\cdot\rangle\rangle$ means the thermal average. In our case, $Q$ is the twist operator

$$
Q(t)=\sum_{\sigma= \pm 1} \int d^{3} r \psi_{\sigma}^{\dagger}(t, \boldsymbol{r}) z L_{z} \psi_{\sigma}(t, \boldsymbol{r}) .
$$

The field operator $\psi$ can be expressed in terms of quasiparticle creation and annihilation operators $b^{\dagger}$ and $b$ as follows:

$$
\psi_{\sigma}(t, \boldsymbol{r})=\sum_{n l m}\left[b_{n l m \sigma} u_{n l m}(\boldsymbol{r}) e^{i E_{n l} t}-\sigma b_{n l m-\sigma}^{\dagger} v_{n l m}^{*}(\boldsymbol{r}) e^{-i E_{n l} t}\right] .
$$

Separating the radial and angular dependence of the wave functions, $\quad u_{n l m}(\boldsymbol{r})=u_{n l}(r) Y_{l m}(\theta, \phi) \quad$ and $\quad v_{n l m}(\boldsymbol{r})$ $=v_{n l}(r) Y_{l m}(\theta, \phi)$, one obtains after a straightforward but tedious calculation the following result:

$$
\begin{aligned}
\Pi_{0}(\omega)= & 2 \sum_{n n^{\prime} l^{\prime} m} m^{2}\left|\int d \Omega Y_{l m}^{*}(\theta, \phi) \cos \theta Y_{l^{\prime} m}(\theta, \phi)\right|^{2} \times\left[\frac { ( E _ { n ^ { \prime } l ^ { \prime } } + E _ { n l } ) [ 1 - f ( E _ { n l } ) - f ( E _ { n ^ { \prime } l ^ { \prime } } ) ] } { ( \omega + i \eta ) ^ { 2 } - ( E _ { n ^ { \prime } l ^ { \prime } } + E _ { n l } ) ^ { 2 } } \left(\int _ { 0 } ^ { \infty } d r r ^ { 3 } \left[u_{n l}(r) v_{n^{\prime} l^{\prime}}(r)\right.\right.\right. \\
& \left.\left.\left.-v_{n l}(r) u_{n^{\prime} l^{\prime}}(r)\right]\right)^{2}+\frac{\left(E_{n^{\prime} l^{\prime}}-E_{n l}\right)\left[f\left(E_{n l}\right)-f\left(E_{n^{\prime} l^{\prime}}\right)\right]}{(\omega+i \eta)^{2}-\left(E_{n^{\prime} l^{\prime}}-E_{n l}\right)^{2}}\left(\int_{0}^{\infty} d r r^{3}\left[u_{n l}(r) u_{n^{\prime} l^{\prime}}(r)+v_{n l}(r) v_{n^{\prime} l^{\prime}}(r)\right]\right)^{2}\right] .
\end{aligned}
$$

In deriving this formula, we have used the anticommutation relations between the operators $b$ and $b^{\dagger}\left[\left\{b_{\alpha}, b_{\beta}\right\}=\left\{b_{\alpha}^{\dagger}, b_{\beta}^{\dagger}\right\}\right.$ $\left.=0, \quad\left\{b_{\alpha}, b_{\beta}^{\dagger}\right\}=\delta_{\alpha \beta}\right]$ as well as the relation $\left\langle\left\langle b_{\alpha}^{\dagger} b_{\beta}\right\rangle\right\rangle$ $=f\left(E_{\alpha}\right) \delta_{\alpha \beta}$, where $f$ denotes the Fermi distribution function, 
$f(E)=1 /\left(e^{E / T}+1\right)$. Note that the relative signs appearing in the radial integrals in Eq. (5) are different from those obtained, e.g., for the case when $Q$ is a multipole operator as in Ref. [8]. The reason is that the twist operator is odd under time reversal, i.e., $\int d^{3} r f^{*}(\boldsymbol{r}) z L_{z} g(\boldsymbol{r})=-\left[\int d^{3} r g^{*}(\boldsymbol{r}) z L_{z} f(\boldsymbol{r})\right]^{*}$. The angular matrix element in Eq. (5) can be computed explicitly, with the simple result

$$
\begin{gathered}
\sum_{m} m^{2}\left|\int d \Omega Y_{l m}^{*}(\theta, \phi) \cos \theta Y_{l^{\prime} m}(\theta, \phi)\right|^{2} \\
\quad= \begin{cases}\frac{\left(l^{\prime}-1\right) l^{\prime}\left(l^{\prime}+1\right)}{15} & \text { if } l^{\prime}=l+1, \\
\frac{(l-1) l(l+1)}{15} & \text { if } l=l^{\prime}+1, \\
0 & \text { otherwise. }\end{cases}
\end{gathered}
$$

Therefore the numerical task of calculating $\Pi_{0}$ reduces essentially to calculating the radial integrals and the triple sum over $n, n^{\prime}$, and $l$.

In general it is not sufficient to calculate the free quasiparticle response $\Pi_{0}$. Rather one has to calculate the quasiparticle random-phase approximation (QRPA) response, which accounts for correlations with the quantum numbers corresponding to the excitation under consideration in the ground state. However, because of the particular form of the interaction used here, it is clear that there cannot be any ground-state correlations with the quantum numbers of the twist mode $\left(J^{P}=2^{-}\right)$. Therefore the QRPA response function $\Pi$ is just equal to the free quasiparticle response function $\Pi_{0}$ [18]. In this sense the situation for trapped atoms is different from that in nuclei, where the spin-orbit part of the interaction leads to a (small) change of the twist response function [14], e.g., through the coupling between the twist mode and the spin-flip mode, which is excited by the operator $(\boldsymbol{r}$ $\otimes \boldsymbol{\sigma})_{20}$.

In the remaining part of this article we will show numerical results for the strength function $S(\omega)=-\operatorname{Im} \Pi(\omega) / \pi$ which we calculate from Eq. (5) with a finite width $\eta$ for each peak.

\section{NORMAL PHASE}

Let us first look at the normal phase of the system at zero temperature, i.e., we artificially put $\Delta=0$ in Eq. (1). For this situation, there exist microscopic descriptions of the twist mode in nuclei $[14,15]$ and in metal clusters [18]. However, for the twist mode in trapped atomic gases there exists only a calculation [19] following the fluid-dynamical approach developed by Holzwarth and Eckart for the nuclear case [13]. This fluid-dynamical approach allows one to predict the twist frequency, but it cannot answer the question if the twist mode as a collective motion exists at all [13].

In the case of a noninteracting $\mathrm{HO}$, it is straightforward to show that the operator $z L_{z}$ excites only transitions with $\omega$ $=1$ (in units of $\hbar \Omega$ ). Therefore, in the noninteracting HO, the total strength is concentrated at $\omega=1$. If now the Hartree potential $W$ is switched on, two effects are to be expected.

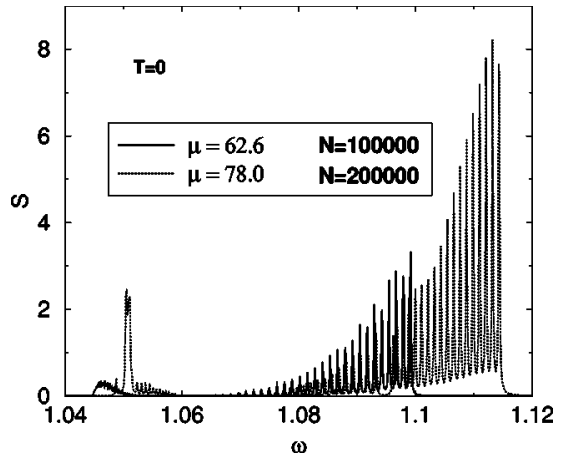

FIG. 1. Strength function $S(\omega)\left(\times 10^{9}\right.$; in HO units $)$ for the twist mode in a gas of $10^{5}$ (solid line) and $2 \times 10^{5}$ (dashed line) trapped ${ }^{6} \mathrm{Li}$ atoms at zero temperature without pairing $(\Delta=0)$ as a function of the frequency $\omega$ (in units of the trapping frequency $\Omega$ ).

(a) The energy difference between neighboring shells becomes larger (smaller) in the case of an attractive (repulsive) interaction. Therefore, the twist frequency will be shifted upward (downward). This effect has been described quantitatively within the fluid-dynamical approach [19].

(b) The degeneracy of states with different $l$ is lifted, and we therefore expect a fragmentation of the strength of the twist mode into many particle-hole states corresponding to transitions $n, l \rightarrow n, l+1$ and $n, l \rightarrow n+1, l-1$ [remember that for given quantum numbers $n$ and $l$, the number of $\mathrm{HO}$ quanta is $2(n-1)+l]$.

Both effects can be observed in Fig. 1, where we display the strength function $S(\omega)$ of the twist mode as a function of the excitation energy for two systems of ${ }^{6} \mathrm{Li}$ with different numbers of atoms (scattering length $a=-2160 a_{0}$ [25], where $a_{0}$ is the Bohr radius) in a trap with a frequency of $\Omega=2 \pi$ $\times 144 \mathrm{~Hz}$, corresponding to a coupling constant $g=-0.4$ in HO units. In order to show the fragmentation of the mode, we display the response function in a small energy interval (containing $100 \%$ of the total strength) with a very high resolution $\left(\eta=10^{-4}\right)$. Let us first look at the result corresponding to $10^{5}$ particles in the trap $\left(5 \times 10^{4}\right.$ particles per spin state, chemical potential $\mu=62.6$ ). One can clearly see that the average frequency is higher than 1 and that the strength is fragmented into two series of peaks, corresponding to the two series of transitions mentioned above under (b). With $2 \times 10^{5}$ particles $\left(10^{5}\right.$ particles per spin state, $\left.\mu=78.0\right)$, the Hartree field is stronger and therefore both effects, fragmentation and shift of the average frequency, are enhanced.

The difference shown by the strength at low frequencies of the systems containing $10^{5}$ and $2 \times 10^{5}$ particles is related to the different single-particle spectra of these two systems. In the case of $10^{5}$ particles, the Hartree field $W$ breaks the accidental degeneracy of the noninteracting HO singleparticle levels, but the different HO major shells are still separated. However, when the number of particles in the trap grows, the Hartree field becomes strong enough to mix different $\mathrm{HO}$ major shells. This leads more or less accidentally to the fact that in the case of $2 \times 10^{5}$ particles the transition energies of the series $n, l \rightarrow n+1, l-1$ with $2(n-1)+l=82$ (the major shell number 82 is the last one lying completely below the Fermi level) are almost degenerate at $\omega \approx 1.05$. 
In order to compare our results quantitatively with the predictions obtained within the fluid-dynamical approach, we define an average frequency according to

$$
\omega_{a v}=\frac{\int_{0}^{\infty} d \omega \omega S(\omega)}{\int_{0}^{\infty} d \omega S(\omega)} .
$$

For both cases considered here, this average frequency is in perfect agreement with the frequency $\omega_{f d}$ predicted in Ref. [19] in the framework of the fluid-dynamical approach: for $N=10^{5}$ atoms, $\omega_{a v}=1.088$ and $\omega_{f d}=1.087$, and for $N=2$ $\times 10^{5}$ atoms, $\omega_{a v}=1.100$ and $\omega_{f d}=1.101$. It should also be emphasized that the width of the interval over which the strength is distributed is very narrow compared with the average frequency of the twist mode. It is therefore justified to speak about a collective excitation.

\section{SUPERFLUID PHASE}

Let us now consider the superfluid case. It is interesting to analyze how the properties of the twist mode are modified when pairing correlations are taken into account in the calculations and the full HFB equations are solved. We will show that the structure of the strength function and the collectivity of the twist mode are strongly affected by pairing correlations and we will study this dependence at different temperatures for two systems with different numbers of atoms.

We set the coupling constant $g$ equal to -1 in $\mathrm{HO}$ units. For ${ }^{6} \mathrm{Li}$ atoms with a scattering length $a=-2160 a_{0}$ this corresponds to a trapping frequency of $\omega=2 \pi \times 817 \mathrm{~Hz}$. (We chose a stronger coupling than in the previous section in order to be able to study the case of strong pairing, which would be possible only for extremely large numbers of particles if $g=-0.4$.) We shall consider two cases for the trapped gas: (a) a small system with around 1800 atoms (weakpairing regime, $\Delta<\hbar \Omega$ ); (b) a big system with around 3.6 $\times 10^{4}$ atoms (strong-pairing regime, $\Delta>\hbar \Omega$ ). For both cases we will take into account different temperatures and analyze how the twist mode evolves when the critical temperature $T_{c}$ of the phase transition is approached and crossed.

Before passing to consider the two cases we would like to mention that in atomic nuclei, which are the only systems for which the twist mode has been observed so far, one is always in the weak-pairing regime, the relation $\Delta<\hbar \Omega$ being always satisfied.

(a) Weak-pairing regime. The chemical potential $\mu$ in this case is chosen equal to 16 . We show in Fig. 2 the strength function for three values of the temperature: $T=0$ (top), 0.13 (middle), and 0.15 (bottom). The three cases correspond to values of the pairing field in the center of the trap of $\Delta(r$ $=0)=0.67,0.26$, and 0 , respectively (in $\mathrm{HO}$ units). In the last case (bottom of the figure) the gas is in the normal phase: we observe that in the normal phase the strength function is concentrated at about $\omega=1.12$ (this is slightly higher than in Fig. 1 because of the stronger coupling, which leads to a

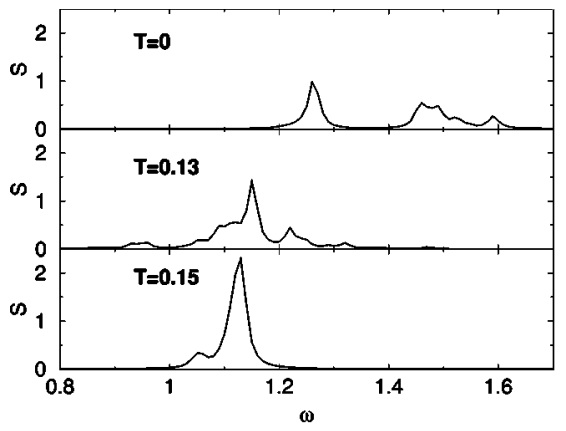

FIG. 2. Strength function $S(\omega)\left(\times 10^{5}\right.$; in $\mathrm{HO}$ units $)$ for the twist mode in a system with about 1800 atoms of ${ }^{6} \mathrm{Li}$ at $T=0$ (top), 0.13 (middle), and 0.15 (bottom); $\omega$ and $T$ in units of $\Omega$ and $\hbar \Omega / k_{B}$, respectively.

stronger Hartree field). If we lower the temperature, the superfluid transition takes place; the effect on the strength function is to push its structure toward higher values of the energy. Qualitatively this can be understood by replacing the single-particle energies $\epsilon_{n l}$ by the quasiparticle energies $E_{n l}$ $\approx \sqrt{\left(\epsilon_{n l}-\mu\right)^{2}+\Delta^{2}}$, where $\Delta$ denotes the average matrix element of the pairing field at the Fermi surface. Neglecting the effect of the Hartree field for the moment, one obtains in this way a shift of the twist frequency from 1 to a higher value which lies between $\sqrt{1+4 \Delta^{2}}$ and $\Delta+\sqrt{1+\Delta^{2}}$. To see this, let us consider two limiting cases. If the chemical potential lies exactly on a single-particle level (half-filled shell), $\mu=N_{F}$ $+3 / 2$, a transition of the type $N_{F} \rightarrow N_{F}+1$, for example, corresponds to the creation of two quasiparticles with energies $E_{N_{F}}=\Delta$ and $E_{N_{F^{+}}+1}=\sqrt{1+\Delta^{2}}$. In the other limiting case, the chemical potential lies between two single-particle levels (closed shell), $\mu=N_{F}+2$, and the twist mode corresponds to the excitation of two quasiparticles having each the energy $E_{N_{F}}=E_{N_{F}+1}=\sqrt{1 / 4+\Delta^{2}}$.

Moreover, as one can also observe in Fig. 2, the excitation mode becomes less collective and, due to pairing, more and more damped and fragmented if one goes from $T=0.13$ to 0 . In the latter case pairing correlations are more intense and the loss of collectivity and the Landau damping are consequently more important. A similar Landau damping effect due to superfluidity has been found in Ref. [8] for the spindipole mode in the weak-pairing regime.

Another interesting effect to notice is the strength below $\omega=1$ which appears below $T_{c}$ but disappears at $T=0$. Obviously this effect is due to the second term in Eq. (5), which is equal to zero at $T=0$.

(b) Strong pairing regime. Let us consider now the case with about $3.6 \times 10^{4}$ atoms $(\mu=40)$. We present in Fig. 3 the strength function at four temperatures: $T=0,2,5$, and 6.5 (from top to bottom). In the four cases the central values of the pairing field are $\Delta(r=0)=12.7,12.5,9.6$, and 0 , respectively. In the latter case (normal phase) we observe a unique peak centered at about $\omega=1.2$. Again, the energy is higher with respect to Fig. 1 and with respect to the case (a) (Fig. 2) due to the stronger coupling. A fragmented structure with a very low strength exists in the energy region from $\omega=2$ up to 4. When we lower the temperature, we cross the superfluid transition (see upper panel of Fig. 4). At $T=5$ the system is 

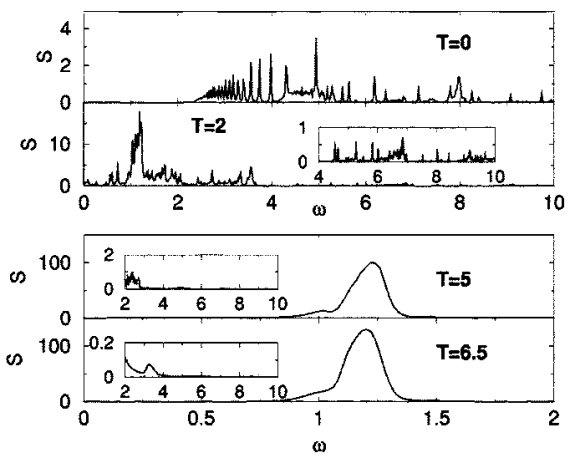

FIG. 3. Strength function $S(\omega)\left(\times 10^{5}\right.$; in $\mathrm{HO}$ units $)$ in a gas with about $3.6 \times 10^{4}$ atoms at four different temperatures (from top to bottom): $T=0,2,5$, and 6.5 ( $\omega$ and $T$ in units of $\Omega$ and $\hbar \Omega / k_{B}$, respectively).

superfluid: we observe that the main peak still exists, even if the excitation mode is less collective than in the normal phase case. Also, the fragmented structure toward $\omega=2-3$ gets more strength than in the previous case. The fragmentation becomes much stronger and extends up to $\omega=10$ when the temperature is lowered further, as can be seen in the case $T=2$. However, the peak at about $\omega=1.2$ is still visible. Finally, if we look at the $T=0$ case, where the pairing correlations are the strongest, we observe that the main collective peak completely disappears, while a very fragmented structure with a low strength remains in the energy region between $\omega=2$ and 10 . We can thus conclude that at $T=0$ the collective twist mode does not exist any more. The same conclusion has been drawn in Ref. [8] for the spin-dipole mode in the strong pairing regime.

Actually, once the irrotational flow limit (strong pairing) is reached [26,27], the superfluid current has an irrotational velocity field, and the only possible excitations of the superfluid are density-fluctuation modes. In the language of a twofluid model, all the other excitations of the gas, such as the twist and the spin-dipole modes, have to be related to its normal component, as was discussed in Refs. [28,29]. When

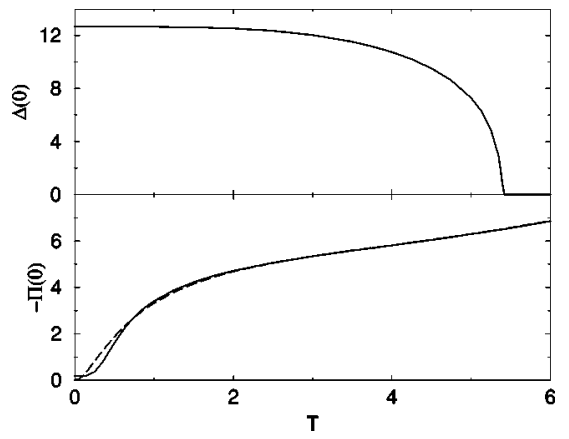

FIG. 4. Temperature dependence of the gap in the center of the trap, $\Delta(0)$ (top; $\Delta$ and $T$ in units of $\hbar \Omega$ and $\hbar \Omega / k_{B}$, respectively), and of the static response function $-\Pi(0)$ (bottom; $\times 10^{-6}$ in $\mathrm{HO}$ units), which is equal to twice the inverse-energy weighted sum rule, for a gas with $3.6 \times 10^{4}$ atoms. In the lower figure, we show for comparison the result of the HFB calculation (solid line) together with the result obtained within the two-fluid model, Eq. (9) (dashed line). one decreases the temperature below $T_{c}$, the number of "normal" quasiparticles is reduced and therefore the strength of the twist mode becomes smaller. On the other side, the energy spectrum of the normal quasiparticles is modified, leading to a destruction of coherence between quasiparticles moving in the same direction [28]. It follows that the mode is more and more damped when one approaches $T=0$. Obviously, this effect will strongly depend on the strength of pairing correlations, and this is why it is more important in the strong-pairing regime.

Let us now discuss the relationship between the strength of the twist response function and the normal-fluid component of the system in a more quantitative way. To that end we consider the inverse-energy weighted sum rule, which is proportional to the real part of the response function at $\omega=0$ :

$$
\int_{0}^{\infty} d \omega \frac{S(\omega)}{\omega}=-\frac{1}{2} \Pi(0) .
$$

Within the two-fluid model it can be shown explicitly (see the Appendix) that this quantity is related to the density of the normal-fluid component of the system, $\rho_{n}$, by

$$
\Pi(0)=-\frac{8 \pi}{15} \int_{0}^{\infty} d r r^{6} \rho_{n}(r) .
$$

In Fig. 4 (bottom) we show numerical results for the dependence of the sum rule on temperature. The solid line represents the full HFB calculation, while the dashed line corresponds to Eq. (9). The agreement is very satisfactory except at extremely low temperature, where quantum finite-size effects (corrections in $\hbar \omega / \Delta$, see Ref. [27]) lead to a nonvanishing value of the sum rule, whereas the two-fluid model predicts that the sum rule should go to zero at zero temperature because of the vanishing normal-fluid component. However, the overall good agreement confirms our interpretation that only the normal-fluid part of the system participates in the twist motion. In order to recognize more easily the regions where the gas is superfluid and normal, and to observe how pairing correlations decrease by increasing the temperature, we also plot in Fig. 4 (top) the value of the gap at the center of the trap, $\Delta(0)$. Note that the temperature dependence of $\Pi(0)$ differs considerably from that of $\Delta(0)$.

To conclude this section, we remark that our approach is only valid in the regime where collisions between atoms can be neglected. Following Ref. [7], this means that the mean time between collisions, $\tau=\rho a^{2} v_{F}\left(T / \epsilon_{F}\right)^{2}$ (where $\rho, v_{F}$, and $\epsilon_{F}$ are the density, Fermi velocity, and Fermi energy, respectively), must be much larger than the oscillation period in the trapping potential, $2 \pi / \Omega$. Expressed in HO units, we obtain $\Omega \tau /(2 \pi)=6 \pi^{3} /(g T)^{2}$. In the case of $T=6.5$ this ratio gives still 4.4 , i.e., an atom performs more than four oscillations before it collides with another one. Consequently, all cases we considered are well in the collisionless regime.

\section{SUMMARY AND OUTLOOK}

In this article we have studied the twist mode of an atomic Fermi gas trapped by a spherical harmonic potential in the 
normal and in the superfluid phase. The ground state has been obtained by solving the Bogoliubov-de Gennes equations, using the regularization procedure introduced in Refs. $[23,24]$. The excitations have been treated within the linear response theory. As the zero-range interaction does not couple to the twist operator, we analyzed this excitation by calculating the free quasiparticle response function.

We have analyzed the twist mode without pairing correlations by setting $\Delta=0$ in the Bogoliubov-de Gennes equations. We observed that the strength function is concentrated around an energy higher than $\omega=1$. This shift (with respect to the case of a noninteracting $\mathrm{HO}$ ) is due to the Hartree potential and depends on the sign of the coupling constant $g$. We have also observed a fragmentation of the strength which describes the transitions $n, l \rightarrow n, l+1$ and $n, l \rightarrow n+1, l-1$.

In the case of pairing correlations we have shown that the excitation mode starts losing its collectivity below the critical temperature $T_{c}$. When the temperature is lowered from $T_{c}$ toward $T=0$, the strength function becomes more and more damped and fragmented. In the weak-pairing regime $(\Delta$ $<\hbar \Omega)$ this effect is less pronounced than in the strongpairing regime $(\Delta>\hbar \Omega)$. In the weak-pairing case the collective twist mode still exists at zero temperature. With increasing strength of the pairing correlations, the collective peak is shifted to higher energies, and at the same time it becomes more and more broad and fragmented and its strength decreases. Finally, in the strong-pairing limit it completely disappears at $T=0$. In fact, it can be predicted that the twist mode ceases to exist once the pairing is strong enough for the system to reach its irrotational flow limit $[26,27]$.

It should be pointed out that, in the normal phase, the twist mode can only exist in the collisionless regime, since the restoring force for this collective oscillation comes entirely from the Fermi surface deformation [13]. This means that detecting the twist mode in the normal phase would be a signal that the system is in the collisionless regime. This might be of importance since the evidence for the superfluidity obtained in recent experiments [9] relies on the assumption that the system is in the collisionless regime. The subsequent disappearance of the twist mode at lower temperatures would be a clear signal that the superfluid phase has been reached. Concerning the possibility to excite the twist mode experimentally we refer to Ref. [19].

Recently, the twist mode has been measured in open-shell finite nuclei such as ${ }^{58} \mathrm{Ni}$ [17]. In the existing theoretical studies of the twist mode in nuclei pairing correlations have not been taken into account, i.e., these studies are essentially restricted to closed-shell (magic) nuclei. Although nuclei are in the weak-pairing regime, we think that a theoretical study of the twist mode in nuclei taking into account pairing correlations could be very interesting. Work in this direction is in progress.

\section{ACKNOWLEDGMENTS}

We thank P. Schuck for interesting discussions. One of us (X.V.) acknowledges financial support from DGI and FEDER (Spain) under Grant No. BFM2002-01868 and from DGR (Catalonia) under Grant No. 2001SGR-00064.

\section{APPENDIX: RELATION BETWEEN THE SUM RULE AND THE NORMAL COMPONENT}

In this appendix we will briefly show how a relationship between $\Pi(0)$ and the normal-fluid component of a system with strong pairing ( $\Delta \gg 1$ in $\mathrm{HO}$ units) can be established. A detailed discussion of some of the topics mentioned here can be found in Ref. [27].

As mentioned in Sec. IV, the inverse-energy weighted sum rule is proportional to the response $\Pi(0)$ of the system to a static perturbation with a perturbation Hamiltonian $H_{1}$ $\propto z L_{z}$. By taking the $\hbar \rightarrow 0$ limit of the time-dependent HFB equations, one can derive equations similar to the Vlasov equation for the superfluid phase (in our case, of course, the time dependence does not play any role). The resulting deviation of the Wigner function $\rho(\boldsymbol{r}, \boldsymbol{p})$ from its equilibrium value reads

$$
\rho_{1}(\boldsymbol{r}, \boldsymbol{p})=\left(\frac{d f(E)}{d E}\right)_{E=E(\boldsymbol{r}, \boldsymbol{p})} h_{1}(\boldsymbol{r}, \boldsymbol{p}),
$$

with

$$
E(\boldsymbol{r}, \boldsymbol{p})=\sqrt{[h(\boldsymbol{r}, \boldsymbol{p})]^{2}+\Delta^{2}(\boldsymbol{r})},
$$

where $h(\boldsymbol{r}, \boldsymbol{p})$ and $h_{1}(\boldsymbol{r}, \boldsymbol{p})$ denote the Wigner transforms of $H_{0}+W(\boldsymbol{r})$ and $H_{1}$, respectively. Since $\Pi(0)$ is defined as the expectation value of $z L_{z}$ in the perturbed system, we can write

$$
\Pi(0)=2 \int \frac{d^{3} r d^{3} p}{(2 \pi)^{3}}\left(\frac{d f(E)}{d E}\right)_{E=E(\boldsymbol{r}, \boldsymbol{p})}\left(z x p_{y}-z y p_{x}\right)^{2} .
$$

Assuming spherical symmetry and a strongly peaked Fermi surface (i.e., $\Delta, T \ll \mu$ ) it is straightforward to derive Eq. (9), where

$$
\rho_{n}(r)=\rho(r) \int d \xi\left(-\frac{d f(E)}{d E}\right)_{E=\sqrt{\xi^{2}+\Delta^{2}(r)}}
$$

is the density of the normal-fluid component within the twofluid model.

Note that the temperature dependence of Eq. (9) is different from that of the number of normal particles, since in Eq. (9) the $r^{6}$ factor weights very strongly the surface of the system, where $\Delta(r)$ is smaller and where consequently the normal-fluid fraction $\rho_{n} / \rho$ is higher than in the center of the trap. 
[1] M. H. Anderson, J. R. Ensher, M. R. Matthews, C. E. Wieman, and E. A. Cornell, Science 269, 198 (1995); K. B. Davis, M.-O. Mewes, M. R. Andrews, N. J. van Druten, D. S. Durfee, D. M. Kurn, and W. Ketterle, Phys. Rev. Lett. 75, 3969 (1995); C. C. Bradley, C. A. Sackett, J. J. Tollett, and R. G. Hulet, ibid. 75, 1687 (1995).

[2] B. De Marco and D. S. Jin, Science 285, 1703 (1999); B. De Marco, S. B. Papp, and D. S. Jin, Phys. Rev. Lett. 86, 5409 (2001); A. G. Truscott, K. E. Strecker, W. I. McAlexander, G. B. Partridge, and R. G. Hulet, Science 291, 2570 (2001); F. Schreck, G. Ferrari, K. L. Corwin, J. Cubizolles, L. Khaykovich, M.-O. Mewes, and C. Salomon, Phys. Rev. A 64, 011402(R) (2001).

[3] K. M. O'Hara, S. L. Hemmer, M. E. Gehm, S. R. Granade, and J. E. Thomas, Science 298, 2179 (2002).

[4] M. Houbiers, R. Ferwerda, H. T. C. Stoof, W. I. McAlexander, C. A. Sackett, and R. G. Hulet, Phys. Rev. A 56, 4864 (1997).

[5] M. A. Baranov and D. S. Petrov, Phys. Rev. A 58, R801 (1998).

[6] G. Bruun, Y. Castin, R. Dum, and K. Burnett, Eur. Phys. J. D 7, 433 (1999).

[7] M. A. Baranov and D. S. Petrov, Phys. Rev. A 62, 041601(R) (2000).

[8] G. M. Bruun and B. R. Mottelson, Phys. Rev. Lett. 87, 270403 (2001)

[9] J. Kinast, S. L. Hemmer, M. E. Gehm, A. Turlapov, and J. E. Thomas, Phys. Rev. Lett. 92, 150402 (2004); M. Bartenstein, A. Altmeyer, S. Riedl, S. Jochim, C. Chin, J. Hecker Denschlag, and R. Grimm, ibid. 92, 203201 (2004).

[10] J. L. Roberts, N. R. Claussen, S. L. Cornish, E. A. Donley, E. A. Cornell, and C. E. Wieman, Phys. Rev. Lett. 86, 4211 (2000).

[11] G. Baym and C. Pethick, The Physics of Liquid and Solid Helium (Wiley, New York, 1978), Pt. II, Chap. 1.

[12] H. Lamb, Proc. London Math. Soc. 13, 189 (1882); G. F. Bertsch, Ann. Phys. (Leipzig) 86, 138 (1979); G. F. Bertsch and R. A. Broglia, Oscillations in Finite Quantum Systems (Cambridge University Press, Cambridge, U.K., 1994).

[13] G. Holzwarth and G. Eckart, Z. Phys. A 283, 219 (1977).

[14] B. Schwesinger, Phys. Rev. C 29, 1475 (1984).

[15] V. Yu. Ponomarev, J. Phys. G 10, L177 (1984).

[16] P. von Neumann-Cosel, F. Neumeyer, S. Nishizaki, V. Yu. Ponomarev, C. Rangacharyulu, B. Reitz, A. Richter, G. Schrieder, D. I. Sober, T. Waindzoch, and J. Wambach, Phys. Rev. Lett. 82, 1105 (1999).

[17] B. Reitz, A. M. van den Berg, D. Frekers, F. Hofmann, M. de Huu, Y. Kalmykov, H. Lenske, P. von Neumann-Cosel, V. Yu. Ponomarev, S. Rakers, A. Richter, G. Schrieder, K. Schweda, J. Wambach, and H. J. Wörtche, Phys. Lett. B 532, 179 (2002).

[18] V. O. Nesterenko, J. R. Marinelli, F. F. de Souza Cruz, W. Kleinig, and P.-G. Reinhard, Phys. Rev. Lett. 85, 3141 (2000).

[19] X. Viñas, R. Roth, P. Schuck, and J. Wambach, Phys. Rev. A 64, 055601 (2001).

[20] B. Schwesinger, K. Pingel, and G. Holzwarth, Nucl. Phys. A 341, 1 (1988).

[21] P. Ring and P. Schuck, The Nuclear Many-Body Problem (Springer-Verlag, Berlin, 1980).

[22] P.-G. de Gennes, Superconductivity of Metals and Alloys (Benjamin, New York, 1966).

[23] A. Bulgac and Y. Yu, Phys. Rev. Lett. 88, 042504 (2002).

[24] M. Grasso and M. Urban, Phys. Rev. A 68, 033610 (2003).

[25] E. R. I. Abraham, W. I. McAlexander, J. M. Gerton, R. G. Hulet, R. Coté, and A. Dalgarno, Phys. Rev. A 55, R3299 (1997).

[26] M. Farine, P. Schuck, and X. Viñas, Phys. Rev. A 62, 013608 (2000).

[27] M. Urban and P. Schuck, Phys. Rev. A 67, 033611 (2003).

[28] A. J. Leggett, Phys. Rev. 140, A1869 (1965); 147, 119 (1966).

[29] O. Betbeder-Matibet and P. Nozières, Ann. Phys. (N.Y.) 51, 392 (1969). 